\title{
Data Processing at Scale
}

This paper was downloaded from TechRxiv (https://www.techrxiv.org).

\section{LICENSE}

CC BY 4.0

SUBMISSION DATE / POSTED DATE

$18-04-2021 / 21-04-2021$

CITATION

Singh, Raju (2021): Data Processing at Scale. TechRxiv. Preprint. https://doi.org/10.36227/techrxiv.14445468.v1

$\mathrm{DOI}$

10.36227/techrxiv.14445468.v1 
Data Processing at Scale.

Name: Raju (rsingh80@asu.edu)

\section{Introduction}

The data generation and collection of data have gone through a series of improvements over the past several years. Now, we observe that both aspects of data (generation and collection) have evolved, it creates another dimension - how to process the data at scale, and how to manage it.

Relational DBMS has been a widely accepted idea behind processing and managing data, but it has its own pros and cons, the constraints on data to prevent integrity violation is seen as a trade-off between performance and management. With the advent in the storage, compute and network technology, we have reliably transited the state of relational database management. It's not yet done. Handling exceptions have been very poor with a single point of failure with traditional DB architecture. However, with distributed systems, it only multiplies the failure points. Failure is expected, and hence the solution for availability is designed around these expected failures. Distributed computing adds functionalities such as performance, availability, and reliability.

But, that's not all. We are living in an era, where we communicate very now and then, through different devices. Not only this, we generate, collect, manage data which are of variant types (mostly unstructured, multi-dimensional, carries lots of noise and bias, etc.). NoSQL DBMS, Apache Spark, and Hadoop come to rescue.

One such area that exemplifies the use of big data is the transportation industry, which can encompass shipping, airline data, trucking, and the context we refer to cabs. NYC taxi data is available in an open-dataset that stores, among other things, geospatial data collected from individual taxis as they navigate the streets of New York City. Processing of geospatial data at this scale is very time-consuming and resource-intensive, as anyone who has used ArcGIS on a large dataset can attest. Distributed and parallel data processing presents an opportunity for faster processing of this type of data. The Apache Spark framework is ideal for this task as it is highly efficient with fast performance times. Additionally, it has libraries and APIs built in that allow it to process SQL queries, which many users are likely to be familiar with given its ubiquity.

In the following report, we demonstrate our approaches to perform hot spot analysis on the NYC Taxi data. Hotzone analysis performs range-join on the rectangle and point, to identify the boundaries from where most pickups happen. Hot-cell analysis uses statistical parameters to identify the zones by also considering time as an additional dimension.

\section{Problem Statement}

\section{Hot Spot Analysis.}

\section{Requirements}

The requirements for the project were:

- Write two User Defined Functions, ST_Contains and ST_Within in SparkSQL.

- Use the two User Defined Functions to perform four spatial queries:

- Range query: Use ST_Contains. Given a query rectangle R and a set of points P, find all

- the points within $\mathrm{R}$.

- Range join query: Use ST_Contains. Given a set of Rectangles R and a set of Points S,

- find all (Point, Rectangle) pairs such that the point is within the rectangle. 
- Distance query: Use ST_Within. Given a point location P and distance D in $\mathrm{km}$, find all

- points that lie within a distance D from P

- Distance join query: Use ST_Within. Given a set of Points S1 and a set of Points S2 and

\section{ST_Contains}

Input: pointString:String, queryRectangle:String

Output: Boolean (true or false) Definition:

Parse the pointString (e.g., "-88.331492,32.324142") and queryRectangle (e.g., "-155.940114,19.081331,$155.618917,19.5307 ")$ to a format that you are comfortable with. Then check whether the queryRectangle fully contains the point. Consider on-boundary point.

\section{ST_Within}

Input: pointString1:String, pointString2:String, distance:Double|

Output: Boolean (true or false)

Definition: You first need to parse the pointString1 (e.g., "-88.331492,32.324142") and pointString2 (e.g., "-88.331492,32.324142") to a format that you are comfortable with. Then check whether the two points are within the given distance. Consider on-boundary point. To simplify the problem, please assume all coordinates are on a planar space and calculate their Euclidean distance.

\section{Design \& Architecture}

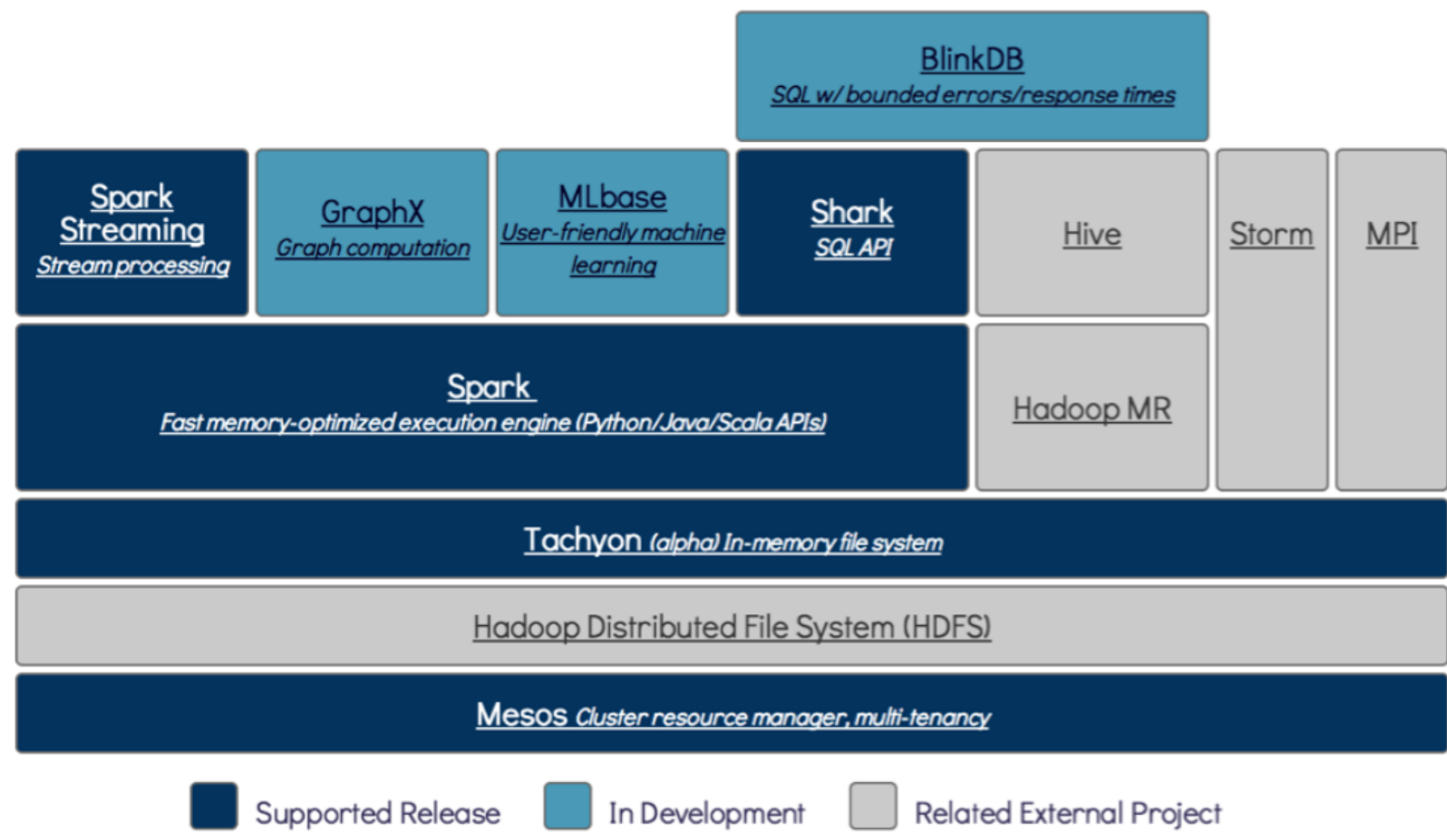

The code will be executed on Apache Spark setup. Apache Spark has a core program component which utilizes Resilient Distributed Databases (RDD). It also has a wide set of libraries:

- MLlib (machine learning)

- $\quad$ SparkSQL

- GraphX (graph analysis) 

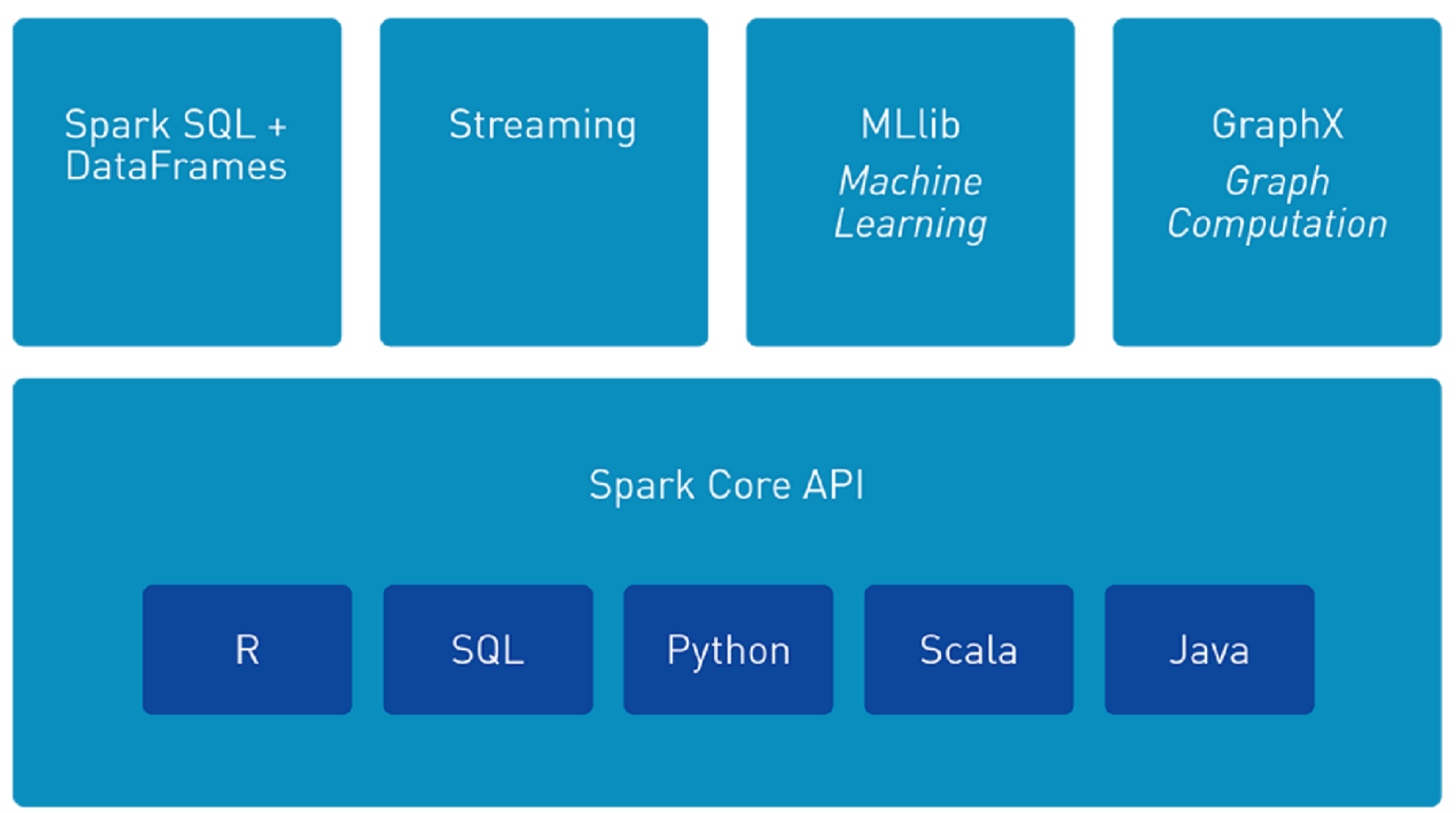

These libraries are built on top of the Spark core and contribute to its ability to perform scalable analysis. SparkSQL runs on top of the Spark core, and interfaces with SQL by using Open DB connectivity or JDBC.

Code were written in Scala language, and used Scala API (Spark flavor). The main task was to modify the exisiting Scala template i.e. SpatialQuery.scala and come up with two new User Defined Functions (as per the requirement). The code design is summarized below:

- The SpatialQuery.scala template imports a SparkSQL session package as:

- import org.apache.spark.sql.SparkSession

- The template defines the object Spatial Query as:

- object SpatialQuery extends App \{\}

- The template defines the following functions and parameters with SpatialQuery:

- def runRangeQuery(spark: SparkSession, arg1: String, $\arg 2:$ String): Long $=\{\}$

- def runRangeJoinQuery(spark: SparkSession, arg1: String, arg2: String): Long = \{ $\}$

- def runDistanceQuery(spark: SparkSession, $\arg 1:$ String, $\arg 2:$ String, $\arg 3:$ String): Long $=\{\}$

- def runDistanceJoinQuery(spark: SparkSession, arg1: String, arg2: String, arg3: String): Long $=\{\}$

- We defined two user_defined functions: st_contains and $c t$ within

- The predefined functions generate DataFrames and then use the user defined functions to determine whether their conditions are met. Results are written to a result Dataframe.

- Once the User Defined Functions are written, the SpatialQuery.scala file is packaged into a .jar file. We used sbt assembly for creating the jar and submitted to SparkSQL spark-submit shell-script. 
select *

from point

where ST_Contains (point._c0, '-155.940114, 19.081331,-155.618917, 19.5307')

\section{Range join query:}

select *

from rectangle, point

where ST Contains (rectangle._c0, point._c0)

\section{Distance query:}

select *

from point

where ST Within(point._c0,' $-88.331492,32.324142^{\prime}, 10$ )

\section{Distance join query:}

\section{select *}

from point p1, point p2

where ST_Within (p1._c0, p2._c0, 10)

\section{Range Query}

The queries in this category restrict Trips with respect to a spatial, temporal, or spatio-temporal point or range. In the examples, the spatial points and ranges are given, respectively, in tables Points and Regions, while temporal points and ranges are given, respectively, in tables Instants and Periods.

Below snippet contains Scala user-defined function (UDF) examples. It shows how to register UDFs, how to invoke UDFs, and caveats regarding evaluation order of subexpressions in Spark SQL.

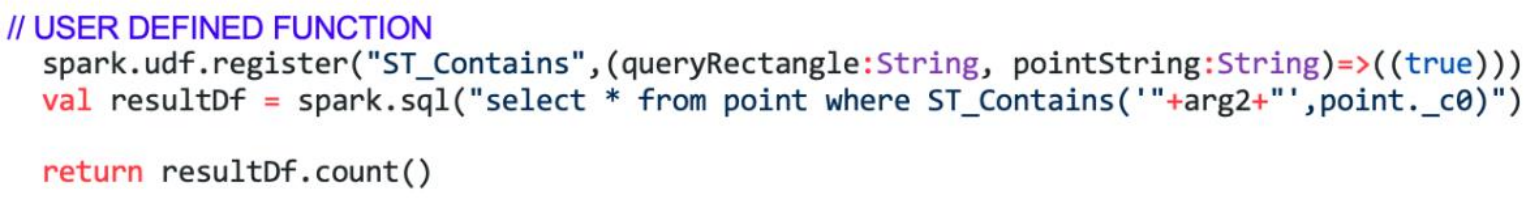


With (set of) points $\mathrm{P}$ and (set of )rectangles R, perform a join on the two datasets and extract the (pts, rect) pairs so that the points is in the rectangle. To determine whether the point belongs to the rectangle or not, use

ST_Contains.

By registering ST_Contains as User Defined Function (UDF), it can be invoked from the Spark SQL Query, resulting in the data frame of point and rectangle pairs.

The following snippet describes registering UDF in Scala API of Spark.

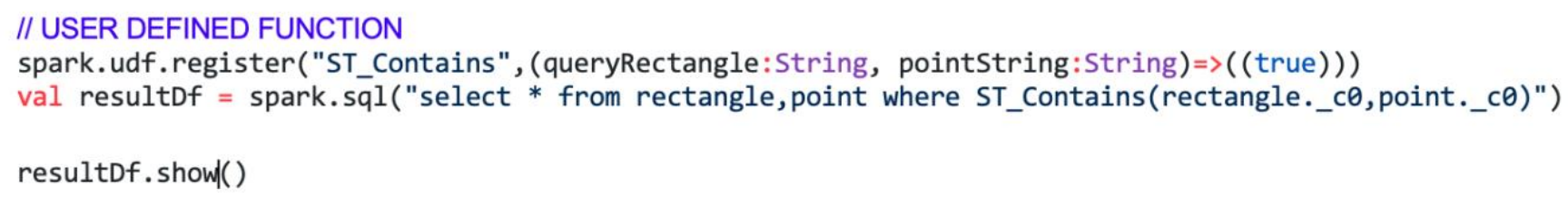

Once the UDF is registered, it is available for use in the SQL API. The following query demonstrate its usage.

select *

from rectangle, point

where ST_Contains (rectangle._c0, point._c0)

\section{Distance query}

The queries in this category deal with either the distance travelled by a single object or the distance between two objects. The complexity of the latter queries depend, on the one hand, on whether the reference objects are static or moving, and on the other, on whether the operation required is either the minimum distance ever or the temporal distance computed at each instant.

User-Defined Functions (aka UDF) is a feature of Spark SQL to define new Column-based functions that extend the vocabulary of Spark SQL's DSL for transforming Datasets.

\section{// USER DEFINED FUNCTION}

spark.udf.register("ST_Within", (pointString1:String, pointString2:String, distance:Double)=>((true)))

val resultDf $=$ spark.sql ("select $*$ from point where ST_Within (point._c0,'"+arg2+" ', "+arg3+") ")

return resultDf.count()

select *

from point

where ST_Within(point._c0,'-88.331492,32.324142',10)

\section{Distance Join Query}

Given a set of points P, perform a join on the two dataset and extract the (point) pairs so that the point is within rectangle. 


\section{Hot Zone}

Given a set of pickup locations $\mathrm{P}$ and a set of zones (rectangles) R, perform range join on the two datasets using ST_Contains and compute the number of points for each cell, which define the hotness of the zone. Once computed return the zones in the ascending order of their rectangle.

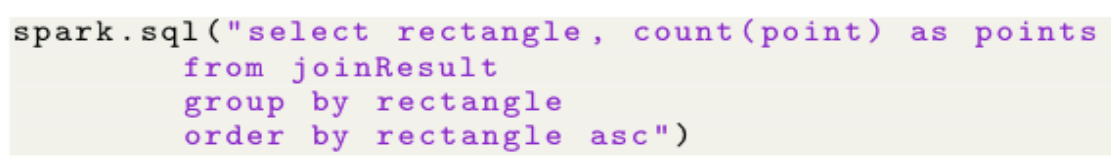

In the above query, joinResult is the output of range join query, on which we group by rectangle to obtain the number of points for each rectangle. The results are then ordered by their rectangle in ascending order. The result RDD is partitioned into multiple partitions, to return as a single partition we use resultDf .repartition(1) function of spark.

Given a list of rectangles and a set of points within each rectangle, determine the hot zone. The hot zone is identified by the number of points in each rectangle. The more the points the hotter the cell is.

The joinResults table view consists of a list of rectangles and points within each of these rectangles. This table view is used to group the rectangles and count the number of points within the rectangle. The resulting query table is ordered by rectangles in ascending order. By default, the results are partitioned into $\mathrm{n}$ tables, and hence the final result needs to be repartitioned into 1 table.

\section{Hot Cell}

Given NYC Taxi Data, considering pickup location, identify statistically significant spatial hot spots by applying spatial statistics. A spatio-temporal cube is formed on by considering the pickup location (latitude and longitude) and time of pickup with given dimensions $(0.01 * 0.01)$. The hot cells are identified using the Getis-Ord $G_{i}{ }^{*}$ statistic also called as z-score.

$G_{i}^{*}$ score is computed using the formula[3] $\frac{\sum_{j=1}^{n} W_{i, j} x_{j}-\bar{X} \sum_{j=1}^{n} W_{i, j}}{S \sqrt{\frac{\left[n \sum_{j=1}^{n} W_{i, j}^{2}-\left(\sum_{j=1}^{n} W_{i, j}\right)^{2}\right]}{n-1}}}$

where $x_{j}$ is the attribute value for cell $j, W_{i, j}$ is the spatial weight between cell $i$ and $j, n$ is equal to the total number

$$
\begin{aligned}
& \text { of cells, } \bar{X}=\frac{\sum_{j=1}^{n} x_{j}}{n} \text {, and } S=\frac{\sum_{j=1}^{n} x_{j}^{2}}{n}-(\bar{X})^{2} \\
& \text { val mean = pickupInfo. } \operatorname{count}() / \text { numCells } / / \mathrm{x} \text {-bar } \\
& \text { val pickupsPerPoint = pickupInfo.groupBy ("x", "y", "z"). count()// } x_{-j} \\
& \text { pickupsPerPoint. createOrReplaceTempView ("tripsFromPoint") } \\
& \text { val sigma_xj_square = spark.sql("select sum(count*count) from tripsFrompoint"). } \\
& \text { first (). get (0). toString. toDouble } \\
& \text { val } \mathrm{S}=\operatorname{math} \text {.sqrt ( (sigma_xj_square/numCells) - math.pow(mean, 2) ) }
\end{aligned}
$$

Computation of $\bar{X}$ and $S$ 


\section{Simplification of Getis-Ord Statistic}

In the given equations, $n$ represents the number of cells in the spatial cube. $W_{i, j}$ is the weight between two cells and is equal to 1 if the cells are neighbors else is 0 . Two cells are neighbors if they are separated by a maximum of 1 unit in any of the three dimensions (latitude, longitude, and time). A cell is also its own neighbor. Thus $\sum_{j=1}^{n} W_{i, j}$ represents number of neighbors $\left(v_{i}\right)$ for each cell and is equal to 8 on corner, 12 on edge, 18 on side, and 27 for all internal cells. $x_{j}$ represents the number of points in a cell. $\sum_{j=1}^{n} W_{i, j} x_{j}$ represents the weighted sum $\left(\sigma_{i}\right)$.

Thus the formula is reduced to

$$
G_{i}{ }^{*}=\frac{\sigma_{i}-\bar{X} v_{i}}{S \sqrt{\frac{\left[n v_{i}-v_{i}^{2}\right]}{n-1}}}
$$

\section{Computation of Neighbours}

Given coordinates of two cells namely $(\mathrm{x} 1, \mathrm{y} 1, \mathrm{z} 1)$ and $(\mathrm{x} 2, \mathrm{y} 2, \mathrm{z} 2)$ the isNeighbour UDF checks whether the cells are neighbors to each other using the following conditions

- $x 1-1<=x 2<=x 1+1$

- $y 1-1<=y 2<=y 1+1$

- $z 1-1<=z 2<=z 1+1$

By using the isNeighbour UDF we compute the weighted sum $\left(\sigma_{i}\right)$ as shown below

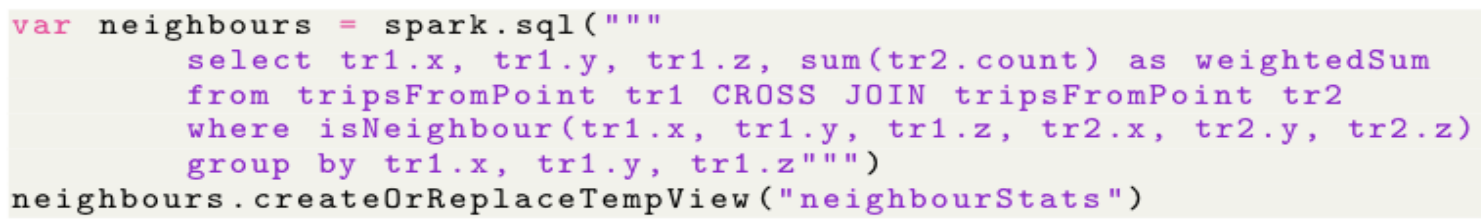

source code to compute weighted_sum $\left(\sigma_{i}\right)$ for each cell

In the above query, we use the table tripsFromPoint, created from the RDD which has the coordinates of cells, pickup time and the number of points belonging to each cell. To compute the weighted sum of each cell, we perform a cross join on the table tripsFromPoint with itself where the cell is a neighbor by using the UDF then perform a group by on each cell to get $\sigma_{i}$ for each cell.

\section{Computation of Getis-Ord Statistic}

As per the above formula, given $\bar{x}, S, n, \sigma_{i}, x, y, z, \min X, \max X, \min Y, \max Y, \min Z, \max Z$, we can compute the $G_{i}{ }^{*}$ score as shown below:

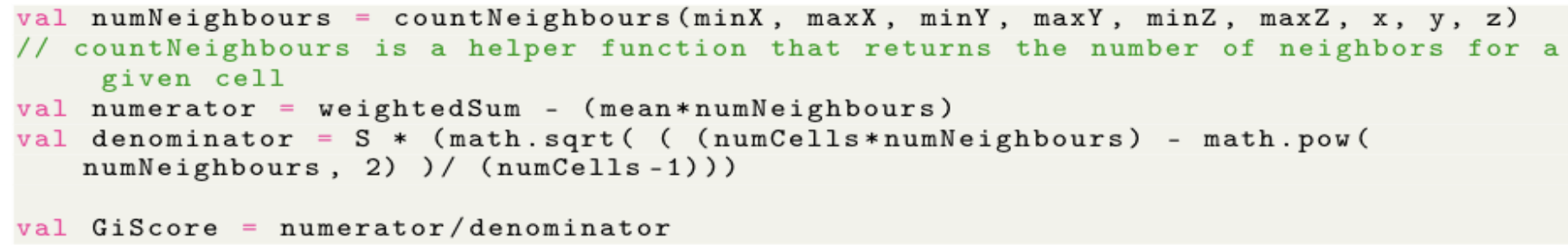

source code for computeGiScore UDF 
Create a table view named "neighbourStats" from the results obtained from neighbours query. We calculate the Gi score for each cell and order it by the giscore in descending order.

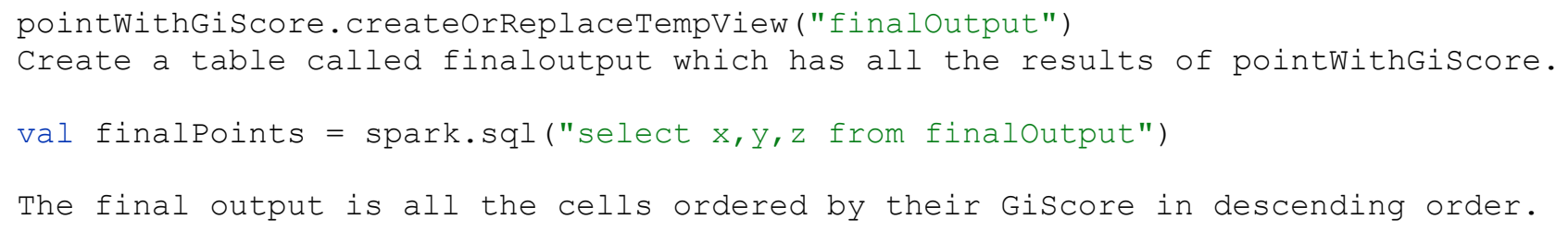

\section{Challenges \& Solutions}

\section{Logical Challenges and Solutions}

We faced majority of challenges during the initial phase of the project, when we discussed on the project requirement and the scope of the project. Each of us contributed the project with inputs and approaches to solve the problem statement. We tracked this using Google doc (meeting notes) for further references.

\section{Technical Challenges and Solutions:}

Most of us were new to the Apache Spark, or initial hurdle was in learning. We did revisit the documentation, videos and tutorial. That helped us to ramp up

\section{Verification, Testing and Validation}

Software verification, testing and validation is an important aspect to establish the software quality. Our code went through this process, where we checked the code quality, code compliance, unit test cases, error handling test cases, expected results etc. This was very important as all of us were learning Scala for the first time, and weren't initially failure with the coding standards. We used online resources as well such as

Since we were all new to scala, we needed a way to test our code as we were writing it. This helped us ensure that we were declaring variables correctly and iterating through arrays correctly. To test while writing our code, we used the online resource, scastie. We compiled the code using sbt-assembly and tested it using spark-submit.

\section{Contribution}

My contribution to the above project can be summarized as:

- Run the scrum meeting.

- Run the weekly meeting.

- Brainstorming on the user journey.

- Timeline discussions, milestones, iterations.

- Technology stack

- Shared lessons learnt on Apache Spark, AWS, Java, SQL with the team.

- Shared the design specifications for the project with the team.

- Code Section: Wrote ST_Within, Distance Query (ST_Within), isNeighbours

- Coded ST_Within section, Distance Query (ST_Within), isNeighbours.

- Merge this code with the main program and run through the test cases.

- Validated the expected output.

- Test and validation of the project.

- Coded test cases to match the user stories.

- Bug fixes, integration errors, troubleshooting development issues.

- Refactoring of the code.

- Manage the Github project repos, updated README.md with installation instructions, test cases etc.

- Github repository management, branching strategy, resolving merge issues, code quality checks, functional and smoke test. 
- Installation instruction, documentation of project report.

I played a critical role in fixing the bug of the program as a whole. Since, each developer was writing their own code, I was in involved and driving discussions around streamlining code, code quality check and enforcing code standards, fixing integration issues and mapping expectations to the initial user journey. I also contributed during the regular sync, created collaboration platform using slack, google doc, for quick sync ups. I used C4model to visualize the diagram

\section{Lessons Learned}

Design practices are an important aspect of software development. During the initial phase of the project, I stressed on the design discussion, before the team can start coding. Before we could start the project, we came up with the framework where we built low level component knowledge, planned to have a system with modular components which are flexible to change. Team insisted to adopt Test Driven Development(TDD) strategy, however I advocated for Behaviour Driven Development (BDD), keeping the customer or user centric need first. Since, I had prior experience of working on this design approach, I had a session with the team where I explain the overview of BDD. I also proposed the layout as:

- define a test set for the unit first;

- make the tests fail;

- then implement the unit;

- finally verify that the implementation of the unit makes the tests succeed.

We adopted and practiced the BDD design protocols. It helped us to finalize the requirement, use cases, design, coding practices etc. This project has given me an opportunity to learn and collaborate more about data management, data analysis and writing code to solve real world problems. As a part of this project, I got an opportunity to learn more about programming language, project management, time management, test case management, collaboration with the team, setting up a development environment locally etc.

Apart from this, I learnt on how to derive the user stories from the requirements, distribute the work among the peer developers, translate it user journey into workable code, run integration specs etc. Working in a team is challenging, yet the learning curve is very steep. For solving real time problems, we need to have collective intelligence and bring more minds.

With regards to the Spark setup, I researched for errors and difficulties which I was facing during the installation. Maintaining proper file structure, making the code idempotent was additional work which improved the code reliability during execution. Scala coding raised initial challenge for me as I wasn't aware of it fully, but it was a learning curve for me once I build the initial understanding.

\section{Interesting Use cases:}

AWS, Hadoop, Spark, Geo-spatial data analysis.

\section{References}

[1]"Spark Class hosted by Stanford ICME", Stanford.edu, 2020. [Online]. Available: https://stanford.edu/ rezab/sparkclass/.

[2]"Behavior-driven development", En.wikipedia.org, 2020. [Online]. Available: https://en.wikipedia.org/wiki?curid=2965801. 